\title{
A Method for Complex Event Detection of Out-of-Order RFID Data Flow
}

Ye DING ${ }^{1,2, a}, H_{e} U^{1,2, b^{*}}$, Ruchuan WANG ${ }^{1,2, c}$ and Weiwei SHEN ${ }^{1,2, d}$

${ }^{1}$ College of Computer, Nanjing University of Posts and Telecommunications, Nanjing 210003, China

2 Jiangsu High Technology Research Key Laboratory for Wireless Sensor Networks, Nanjing, 210003, China

\author{
a1215043121@njupt.edu.cn, ${ }^{*}$ Corresponding author, \\ bxuhe@njupt.edu.cn, cwangrc@njupt.edu.cn, d1214042932@njupt.edu.cn
}

Keywords: RFID; Complex Event Detection; Binary Tree; Out-of-Order

\begin{abstract}
Because RFID events contain features of real-time and mass resistance, there are some issues through the existing complex event detection method for processing, such as inefficient speed and taking up larger memory space. To solve these problems, a complex event detection method based on binary tree is proposed. The algorithm designs an effective binary tree and combines the atomic events into complex events. Analysis shows that the complex event detection method has high efficiency and accuracy in theory and operational feasibility.
\end{abstract}

\section{Introduction}

With the further application and development of Radio frequency identification (RFID) technology, there are large amount of event data[1]. RFID is a communication technology that can recognize specific target by radio signals and read or write data. The connection between the recognition system and the special target don't require mechanical or optical contact.

In the identification, logistics management, security monitoring and other applications areas, it is very important for managing task and making decision to collect real-time information[2]. The RFID application system should include these components[3]: readers, tag, the upper application, as shown in Figure 1. Reader contains the antenna and reader devices, and each reader can identify tag with multiple antennas. The reader can read and write data on its label in the radio signal coverage. Tag includes the chip and the antenna, which has a unique identification ID. The upper application mainly handles data collected by RFID systems.

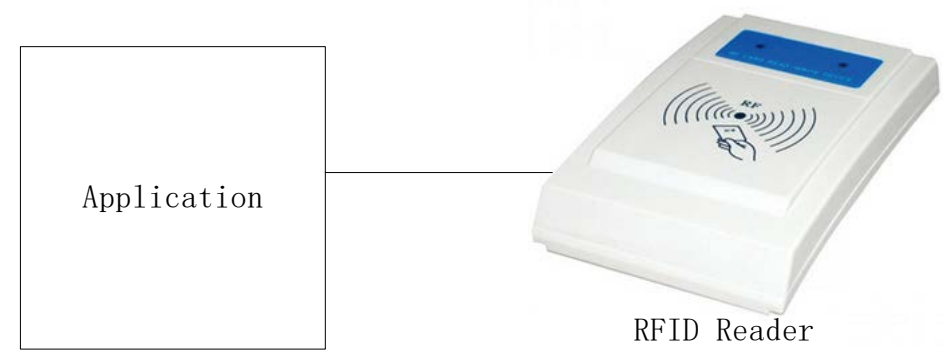

Figure 1. RFID System

\section{Related Work}

However, the atomic events (namely raw data) collected by RFID systems has limited semantics and can't meet the demands in business. Complex event processing[4] (CEP) is a technology for processing large amount of raw data generated by RFID system, which combines a number of events into complex and obtains meaningful value. 
Data stream is the combination of information collected by RFID system, and there is sequence in data stream. Sequence is that Event A and Event B, while their occurrence time is T(A) and T(B). When $\mathrm{T}(\mathrm{A})<\mathrm{T}(\mathrm{B})$, it is supposed to make sure that the Event $\mathrm{A}$ is the output before the Event $\mathrm{B}$.

Literature[5-7] studied the definition and optimization of complex event processing and disorder of events in RFID data stream. These studies assume that complex events are from a single data stream and there are no incomplete event detection. However, in high-density stream of events, it is difficult to promise that the sequence among events is always proper. This paper studies how to efficiently detect the event flow from out-of-order RFID complex event data flow.

\section{Event Model}

Event generated by RFID application systems is a triple $(\mathrm{R}, \mathrm{O}, \mathrm{T})$, where $\mathrm{R}$ represents the identification reader, $\mathrm{O}$ means the identification object, $\mathrm{T}$ stands for the time when the reader reads the object. Event query is defined by declarative query language, which uses SASE[8] to define complex event queries. The whole frame of SASE is shown as follows:

EVENT (event pattern ),

WHERE (qualification),

WITHIN(time window).

EVENT uses event operators (such as SEQ, AND, OR, NOT, etc.) to define the pattern to be detected. WHERE describes the constraints between events. WITHIN specifies a time window in the continuous data stream.

\section{Event Example}

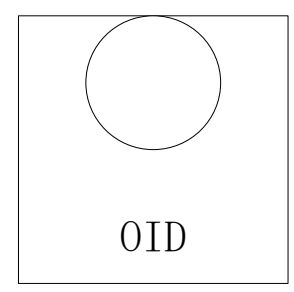

SCENE A

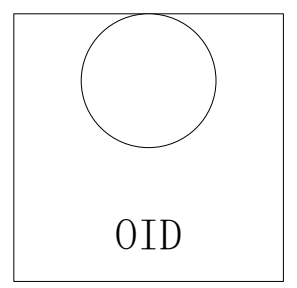

SCENE B

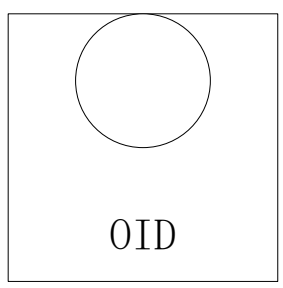

SCENE C

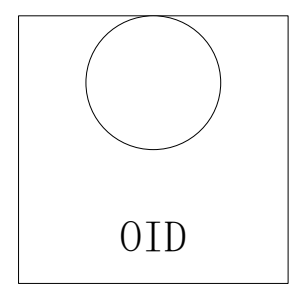

SCENE D

Figure 2. RFID objects used in different scenarios

As shown in Figure 2, objects with the same RFID tag is used in different scenarios. In the ideal case, the order is Scene A, Scene B, Scene C, Scene D. In order to find that the tag is detected by the reader $A, B, C, D$ in sequence within 60 minutes, this paper defines the following query:

EVENT SEQ (A,B,C,D)

WHERE $($ A.OID $=$ B.OID $=$ C.OID = D.OID $)$

WHITIN 60 minutes.

Among the above query, A.OID, B.OID, C.OID, D.OID have the same ID, which represents that the object with the same tag is applied in the different scenarios.

Event query is resolved to non-deterministic finite automata (NFA)[9] for execution, and each state is as an event type in NFA. The transfer between states is corresponding to the constraint detection between events.

\section{Method}

In ideal situation, the event occurs firstly should be delivered to the event handler firstly, that is the event in the events stream occurred in sequence. However, in practice, events may not be arranged in the order of occurrence, which may appear out of order. Disorder will affect the accuracy of complex event detection, thus decisions depend on these results is unreliable. Therefore, it is necessary to process the disorder in the complex event stream. 
In order to deal with out of order events stream, this paper proposes two steps to rectify the effect of the disorder events. Firstly, it is need to modify the original automatic handling mechanism. For detection model SEQ (A, B, C, D), the initial state of the automaton is A, the state transition is in strict accordance with the order of A, B, C, D. However, in the chaos Preface, A, B, C, D can be starting point, there is no sequence limit in the transition of state. Then, the first event received is defined as the head node of a binary tree. In the following events stream, according to time when the event occurs , make the first occurred event as the left subtree, and the later occurred event as the right subtree. Specific steps are as the follows:

Algorithm: Detection method of the out-of-order events

Enter: the event flow Event Stream, query Q.

Output: the result of complex event stream.

1) Read the query $Q$ and produce the corresponding NFA, calculate the sequence which the NFA recognizes the length of sequence and create an empty binary tree;

2) Get new event $E$ from the atomic events;

3) Detecting whether the NFA receive E, if $E$ is accepted then turn to 4), else go to 2);

4) Determine whether there is a binary tree head node, if there is no head node, make the event $\mathrm{E}$ as the head node; if there is, compare the time of occurrence of event $\mathrm{E}$ with the time of the head node. If the event $\mathrm{E}$ is earlier, then compare the left subtree; if the event $\mathrm{E}$ is later, compare the right subtree.

5) Insert the event $\mathrm{E}$ into the binary tree according the time, then turn to 2);

6) When all queried events are added to the binary tree, combine and output the complex event made of all events according to the preorder.

Suppose that the time sequence of occurrence of atomic event in the events stream is $7,9,11,2,3,8$. According to the above algorithm, the binary tree can be drawn as shown in Figure 3:

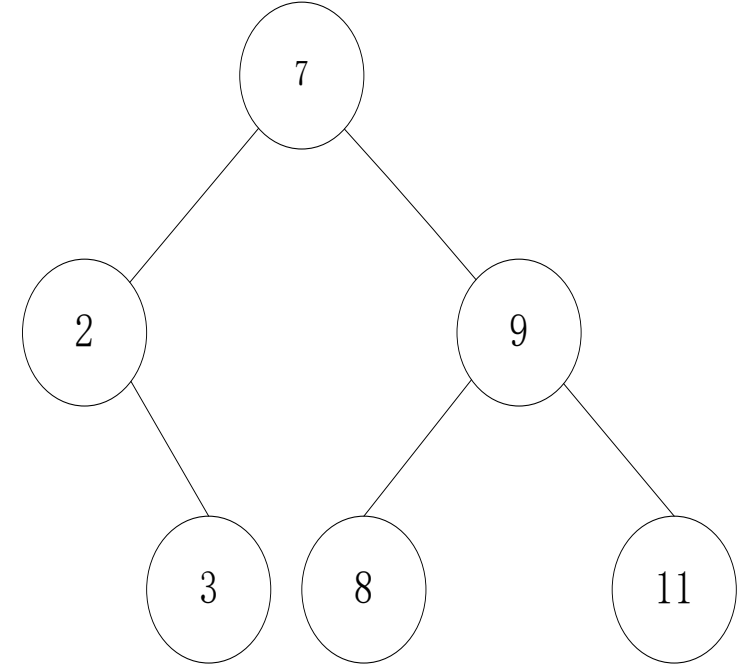

Figure 3. Binary tree occurred by the disorder algorithm

Figure 3 is the binary tree obtained by the above algorithm, and the result is 2,3,7,8,9,11 according to the preorder of the binary tree.

\section{Conclusions}

This paper mainly researches and deals with the RFID event stream and proposes a complex event detection method of the disorder event stream. This method saves the complex event in need through building the binary tree by the algorithm, which also solves the problems carried by the disorder of event stream. Analysis shows that the complex event detection method has high efficiency and accuracy in theory and operational feasibility. In the future, the massive and concurrent performance and real-time system need to be improved in order to support the parallel process in RFID data stream. 


\section{Acknowledgements}

This work was financially supported by the Natural Science Foundation of Jiangsu Province, China(Grant No. BK20140886, No. BK20140888), China Postdoctoral Science Foundation(Grant No. 2014M561696, No. 2014M551636), Natural Science Foundation of the Jiangsu Higher Education Institutions of China(Grant No. 14KJB520030), Jiangsu Planned Projects for Postdoctoral Research Funds(Grant No. 1401005B, No. 1302090B), Jiangsu Graduate Scientific Research and Innovation Projects (SJLX15_0381)and NUPTSF(Grant No. NY213034, No. NY214060 and No. NY214061).

\section{References}

[1] G. Cugola and A. Margara, "Processing flows of information: From data stream to complex event processing", ACM Computing Surveys (CSUR). DOI: 10.1145/2187671.2187677, vol.44, no.3, pp.15,(2012).

[2] H. J. Yazici, "An exploratory analysis of hospital perspectives on real time information requirements and perceived benefits of RFID technology for future adoption", International Journal of Information Management, vol.34, no.5, pp.603-621,(2014).

[3] A. Akbari, S. Mirshahi and M. Hashemipour, "Application of RFID system for the process control of distributed manufacturing system",in 2015 IEEE 28th Canadian Conference on Electrical and Computer Engineering (CCECE),pp.497-501,( 2015).

[4] C. C. Aggarwal, Managing and mining sensor data, Springer Science \& Business Media, 2013.

[5] S. Peng, H. Liu, X. Guo and J. He, "State-based event detection optimization for complex event processing", Sensors \& Transducers, vol.164, no.2, pp.242,(2014).

[6] J. Wang, L. Cheng and J. Liu, "A complex event detection method for multi-probability RFID event stream", Journal of Software, vol.9, no.4, pp.834-840,(2014).

[7] J. Hu, F. L. Lewis, O. P. Gan, G. H. Phua and L. L. Aw, "Discrete-Event Shop-Floor monitoring system in RFID-Enabled manufacturing", IEEE Transactions on Industrial Electronics, vol.61, no.12, pp.7083-7091,(2014).

[8] D. Gyllstrom, E. Wu, H. Chae, Y. Diao, P. Stahlberg and G. Anderson, "SASE: Complex event processing over streams",in 3rd Biennial Conference on Innovative Data Systems Research (CIDR),pp.1-5,( 2007).

[9] A. Kumar and A. K. Verma, "Conversion of parallel regular expressions to non-deterministic finite automata using partial derivatives", Chiang Mai Journal of Science, vol.41, no.5.2, pp.1409-1418,(2014). 